\title{
Who would I consider for capsule endoscopy? - Part I
}

\author{
Robert Enns MD
}

$\mathrm{T}_{\mathrm{p}}^{\mathrm{h}}$ he advent of capsule endoscopy (CE) has been changing the paradigms of gastrointestinal (GI) evaluations ever since it was granted approval by the Food and Drug Administration in 2001. Over the past five years, significant changes have occurred in numerous areas, particularly with the assessment of the small intestine. As familiarity and availability have increased, endoscopic indications also seem to have expanded. Additionally, with new technology emerging (ie, Olympus capsule [Olympus Corporation, Tokyo, Japan], double balloon enteroscopy, esophageal and colonic capsules), these changes in management will continue to evolve and educational updates are critical (for physicians, patients and regulatory bodies) to maintain an appropriate high standard of care.

The initial studies $(1,2)$ involving capsule endoscopy demonstrated superiority over the 'standard' push enteroscopy (PE) in both canine and human subjects. These studies concentrated on aspects of obscure GI bleeding (OGIB) which is usually relatively severe, and commonly overt. Since the initial hallmark studies, numerous other studies $(1,3-7)$ have demonstrated efficacy in many other areas.

The key areas to consider for indications for CE include OGIB, inflammatory disorders of the small intestine, polyposis syndromes, clarifying abnormal radiological imaging and chronic pain assessment

Most CEs are performed for OGIB, which is classified into overt and occult bleeding. OGIB is commonly defined as recurrent bleeding without an identifiable source, noted on investigations including esophagogastroduodenoscopy and colonoscopy. Although this definition is adequate in some situations, it fails to include patients with persistent bleeding who in fact may not have recurrent but only ongoing bleeding (either occult or overt). The yield of CE has been demonstrated, in at least one study (8), to be higher in patients with ongoing overt bleeding as opposed to previous episodes of bleeding or iron deficiency anemia.

Whether a PE (with either a dedicated enteroscope or a pediatric colonoscope) is required before CE is a point of heated debate. The proponents of PE argue that the duodenum is poorly visualized by CE and a significant yield is found in PE, thereby saving the capsule resource for patients requiring it. Others argue that clearly CE has a higher yield $(2,7,9)$ in small bowel disorders compared with $\mathrm{PE}$, so $\mathrm{PE}$ is just one more added invasive procedure that adds cost to the overall investigations with an inferior diagnostic yield. In a Canadian setting where CE is infrequently reimbursed and relatively expensive in comparison with $\mathrm{PE}$, I have argued in favour of performing a PE first in the setting of OGIB. With enhanced reimbursement and perhaps decreasing costs of the actual capsule, my stance on this may change in the future.

Selecting patients with OGIB for CE is difficult. We have determined that the only predictors of positive outcomes have been transfusion requirements. Essentially, the more blood a patient requires via transfusions, the more likely a positive outcome will be determined on CE. Additionally, one other study (9) has suggested that patients with ongoing overt bleeding have a higher yield in CE, therefore this is another group that should be targeted to enhance positive outcomes. Chronic iron deficiency with no evidence of bleeding has a low yield on CE, particularly in comparison with other groups noted above. Patients with iron deficiency are now the most common, and perhaps the only group that I would personally order occult blood testing for. The stools are usually recurrently negative, demonstrating no evidence of occult bleeding, and the etiology of the problem is other than OGIB.

The performance of CE on patients who are not deemed to be surgical candidates (because of comorbid conditions) is an interesting problem because in most locations in Canada, surgical therapy is recommended for lesions outside the reach of endoscopic assessment. With the advent of double balloon enteroscopy, this group of patients can theoretically be assessed and possibly treated endoscopically, and thus, no lesion is 'out of reach.' Additionally, from Robert Enns personal experience, it is interesting to note that nonsurgical candidates can be converted to surgical candidates when impressive definitive images of a significant bleeding lesion are noted within the small bowel.

Inflammatory disorders of the small intestine can include Crohn's disease (CD), celiac disease and other less common malabsorptive and infiltrative states (ie, immunodeficiency syndromes, ulcerative jejunoileitis, eosinophilic enteritis, amyloid and Whipple's disease). Although the use of CE has significant potential for diagnosis and monitoring in many of these disorders $(3,10-12)$, at present it is used in my practice only very selectively. Despite the fact that CE clearly provides ideal images of the small intestine (10), inflammatory disorders of the small intestine can usually be diagnosed with more standard, widely available endoscopic and radiological tests. Most of the patients referred for possible CD are in need of a thorough consultation as opposed to more testing. It may be easier in some situations to perform a CE instead of an extended consultation with the patient; however, the history will usually 'point' in the right direction. Despite this and because of increased sensitivity, some studies $(13,14)$ have suggested an economic benefit of performing CE early in the diagnostic algorithm of inflammatory small bowel disorders.

The true value of CE may be in assessing complications and response to treatment of various inflammatory conditions. In the setting of celiac disease, there are several studies $(14,15)$ that confirm benefit, particularly in those patients who have a poor response to therapy or those who are deemed to be suffering from a complication. Additionally, patients with suspected celiac disease and negative or equivocal biopsies (often with positive antibody studies) may benefit from CE.

Similarly in CD, the biggest market may eventually include assessment of mucosal healing. The improvements that have accompanied the patency capsule will add a margin of safety to assessment in CD. Although not extensively used at present, the use of CE to assess response to therapy in inflammatory diseases 
(particularly with newer biological agents) is likely to blossom. A standardized, validated scoring system for ulceration in $\mathrm{CD}$ is still lacking in CE, but studies are ongoing.

Lastly, the benefit of CE in the setting of indeterminate colitis is unclear but evolving. Twenty per cent to $40 \%$ of patients with indeterminate colitis have abnormalities of the small intestine (nonspecific ulceration); however, the significance of this is unclear. With future study perhaps combined with other diagnostics, CE may be determined to assist in selecting appropriate patients for pouch procedures.

Polyposis syndromes are a relatively uncommon condition in comparison with OGIB; however, unlike patients with OGIB, polyposis patients will likely require repeated studies for surveillance purposes. In the setting of familial adenomatous polyposis (FAP), early studies $(16,17)$ suggest various benefits of assessing all patients for small bowel polyps. More recently, it has been recognized that most FAP patients who have small intestinal polyps are accessible to enteroscopy and/or side-viewing endoscopy (where the papilla can also be assessed). In general, patients with FAP who have limited polyp burden in the duodenum (Spigelman stages 0 to 2) without a history of small bowel polyps and no clear cut evidence of distal duodenal disease likely do not require surveillance of the small intestine with capsule studies (18).

Peutz-Jeghers syndrome is another small intestinal polyposis syndrome for which surveillance is recommended. Before CE, barium studies were commonly used, the size of the polyp was estimated and surgery was considered. The advent of CE improves detectability; however, estimation of polyp size is still difficult. Despite this limitation, most experts consider CE to be an improvement for early detection of small bowel polyps in PeutzJeghers syndrome and surveillance is usually recommended, generally every two years.

Data for other polyposis syndromes (both familial and nonfamilial) are lacking because they are much less common. However, in all patients with polyposis syndromes who previously had recommendations for surveillance via barium studies, it seems logical that these will be replaced by the more sensitive capsule study.

Abnormal radiological imaging is becoming more frequent as screening radiological examinations become more popular. Often lesions adherent to or within the small intestine are suspected, but not confirmed, on radiological imaging. In the past, repeat studies may have been suggested in three to six months to clarify the diagnosis. However, with the advent of CE, a more definitive diagnostic test has become available. The most common situation that arises is with computed tomography of the abdomen, as well as small bowel barium studies, where nonspecific thickening or nodularity may be seen in the small intestines that are not accessible through standard endoscopic means. Assessing the area of abnormality via CE has been requested on numerous occasions, usually demonstrating nonspecific findings; however, as the 'gold standard' for the small bowel, a definitive decision on these lesions is critical. The use of CE to assess and confirm radiological abnormalities will increase in the future.

The assessment of chronic pain conditions is a very challenging task. Many gastroenterologists tend to avoid the problem and because these patients tend to be 'desperate' they are referred for $\mathrm{CE}$ studies. The use of $\mathrm{CE}$ as a 'final' investigation before supportive care in chronic abdominal pain has been suggested. However, this has not been proven to be an efficacious or cost-effective method of management. Although abnormalities are determined in a small number of patients, these are often not the cause of their pain, and an incidental finding often simply heightens anxiety as opposed to alleviating it (19). At present, I do not advocate CE in patients with chronic pain syndromes, although the benefit or lack thereof remains to be demonstrated. Future studies, particularly in regard to the ability to reassure the patient after a negative study, are required before recommendations can be forthcoming.

The use of CE in all areas is evolving. Esophageal capsules and colonic capsules will add to the armamentarium of the gastroenterologist. As studies continue in this rapidly developing field, it is paramount that physicians understand the value and limitations of these devices in the assessment of GI disorders. The lack of widespread availability and apparent relative expense should not dissuade physicians from pursuing ideal imaging for their patients.

\section{REFERENCES}

1. Appleyard M, Fireman Z, Glukhovsky A, et al. A randomized trial comparing wireless capsule endoscopy with push enteroscopy for the detection of small-bowel lesions. Gastroenterology 2000;119:1431-8.

2. Lewis BS, Swain P. Capsule endoscopy in the evaluation of patients with suspected small intestinal bleeding: Results of a pilot study. Gastrointest Endosc 2002;56:349-53.

3. Bhinder F, Schneider DR, Farris K, et al. NSAIDs-associated small intestinal ulcers and strictures: Diagnosis by video-capsule enteroscopy. Gastroenterology 2004;122:A345. (Abst)

4. Costamagna G, Shah SK, Riccioni ME, et al. A prospective trial comparing small bowel radiographs and video capsule endoscopy for suspected small bowel disease. Gastroenterology 2002;123:999-1005.

5. Eliakim R, Fischer D, Suissa A, et al. Wireless capsule video endoscopy is a superior diagnostic tool in comparison to barium follow-through and computerized tomography in patients with suspected Crohn's disease. Eur J Gastroenterol Hepatol 2003;15:363-7.

6. Ell C, Remke S, May A, Helou L, Henrich R, Mayer G. The first prospective controlled trial comparing wireless capsule endoscopy with push enteroscopy in chronic gastrointestinal bleeding. Endoscopy 2002;34:685-9.

7. Van Gossum A, Hittelet A, Schmit A, Francois E, Deviere J. A prospective comparative study of push and wireless-capsule enteroscopy in patients with obscure digestive bleeding. Acta Gastroenterol Belg 2003;66:199-205.

8. Pennazio M, Santucci R, Rondonotti E, et al. Outcome of patients with obscure gastrointestinal bleeding after capsule endoscopy: Report of 100 consecutive cases. Gastroenterology 2004;126:643-53.

9. Mylonaki M, Fritscher-Ravens A, Swain P. Wireless capsule endoscopy: A comparison with push enteroscopy in patients with gastroscopy and colonoscopy negative gastrointestinal bleeding. Gut 2003;52:1122-6.

10. Fireman Z, Mahajna E, Broide E, et al. Diagnosing small bowel Crohn's disease with wireless capsule endoscopy. Gut 2003;52:390-2.

11. Liangpunsakul S, Chadalawada V, Rex DK, Maglinte D, Lappas J. Wireless capsule endoscopy detects small bowel ulcers in patients with normal results from state of the art enteroclysis. Am J Gastroenterol 2003;98:1295-8.

12. Schreyer AG, Golder S, Seitz J, Herfarth H. New diagnostic avenues in inflammatory bowel diseases. Capsule endoscopy, magnetic resonance imaging and virtual enteroscopy. Dig Dis 2003;21:129-37.

13. Goldfarb NI, Pizzi LT, Fuhr JP Jr, et al. Diagnosing Crohn's disease: An economic analysis comparing wireless capsule endoscopy with traditional diagnostic procedures. Dis Manag 2004;7:292-304.

14. Petroniene R, Dubcenco E, Baker JP, et al. Given capsule endoscopy in celiac disease: Evaluation of diagnostic accuracy and interobserver agreement. Am J Gastroenterol 2005;100:685-94.

15. Culliford A, Daly J, Diamond B, Rubin M, Green PH. The value of wireless capsule endoscopy in patients with complicated celiac disease. Gastrointest Endosc 2005;62:55-61.

16. Burke CA, Santisi J, Church J, Levinthal G. The utility of capsule endoscopy small bowel surveillance in patients with polyposis. Am J Gastroenterol 2005;100:1498-502.

17. Caspari R, von Falkenhausen M, Krautmacher C, Schild H, Heller J, Sauerbruch T. Comparison of capsule endoscopy and magnetic resonance imaging for the detection of polyps of the small intestine in patients with familial adenomatous polyposis or with Peutz-Jeghers' syndrome. Endoscopy. 2004;36:1054-9.

18. Burke CA, Santisi J, Church J, Levinthal G. The utility of capsule endoscopy small bowel surveillance in patients with polyposis. Am J Gastroenterol 2005;100:1498-502.

19. Bardan E, Nadler M, Chowers Y, Fidder H, Bar-Meir S. Capsule endoscopy for the evaluation of patients with chronic abdominal pain. Endoscopy 2003;35:688-9. 


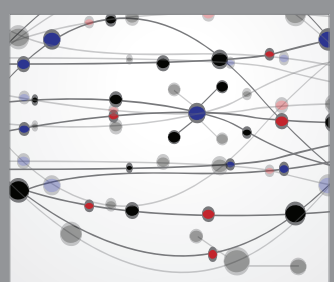

The Scientific World Journal
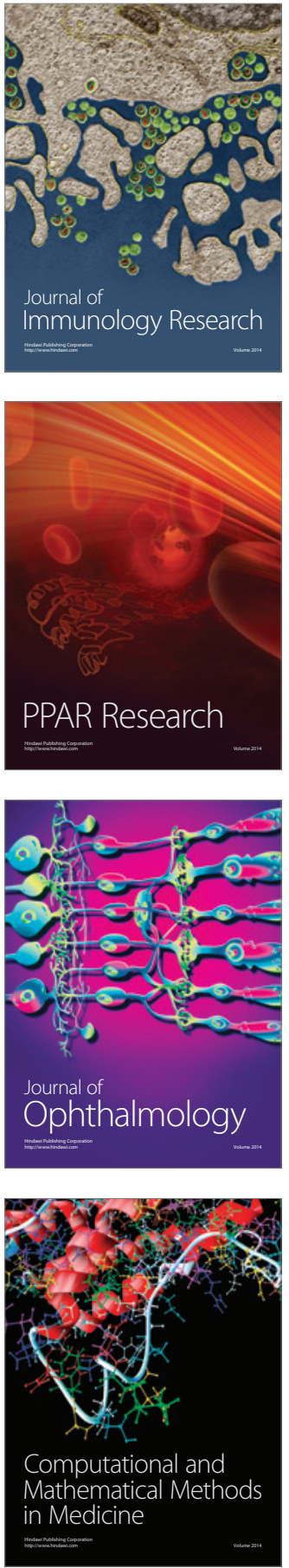

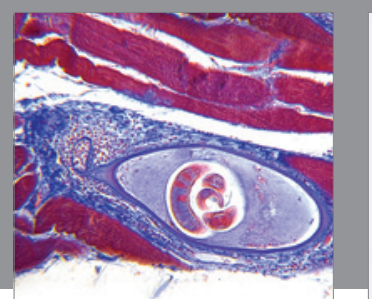

Gastroenterology Research and Practice

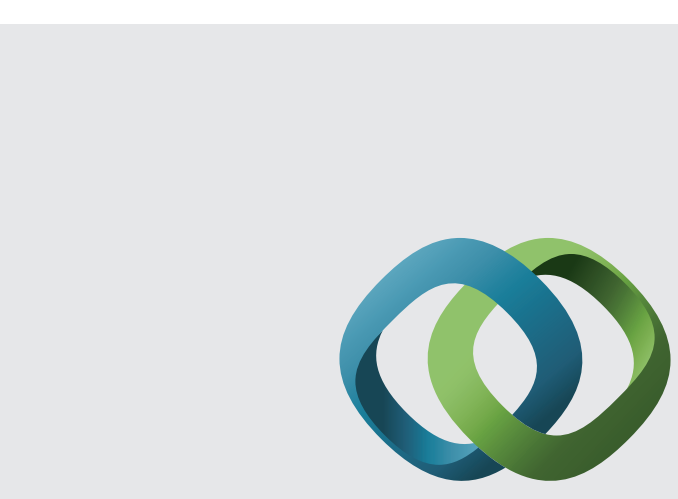

\section{Hindawi}

Submit your manuscripts at

http://www.hindawi.com
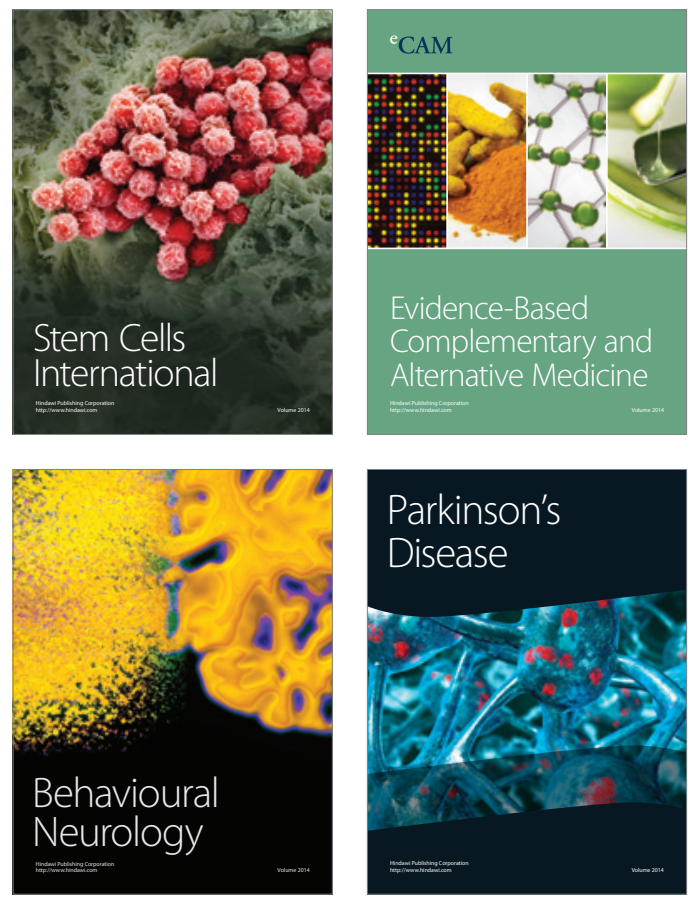
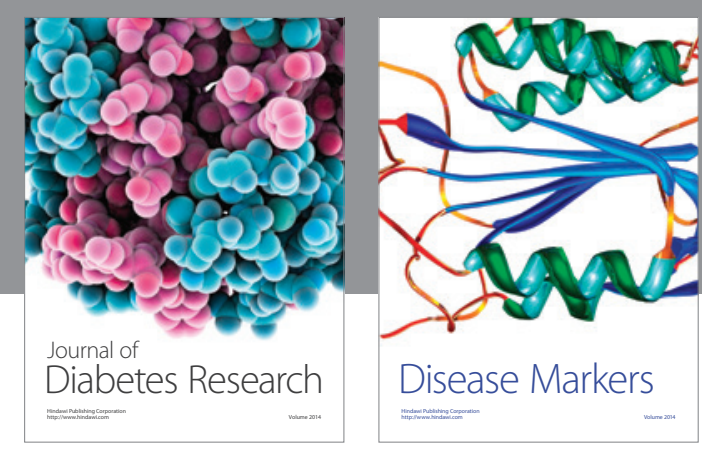

Disease Markers
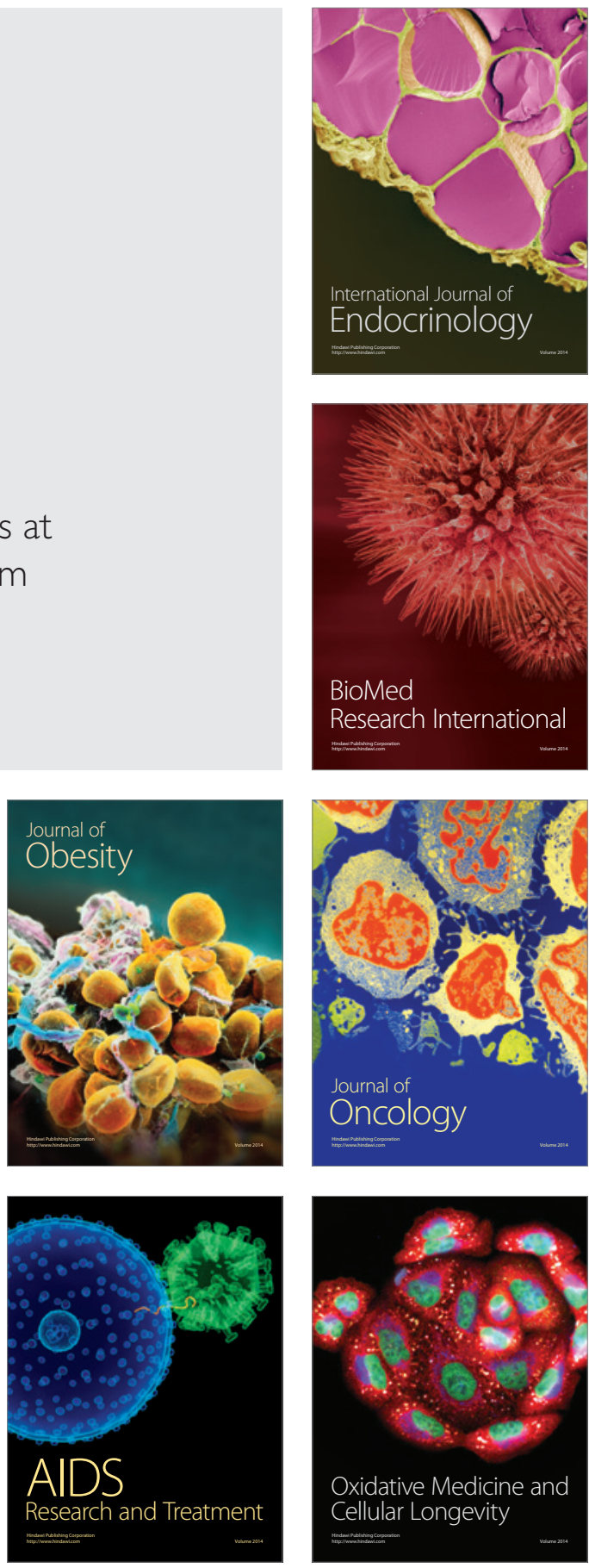